\title{
CNO and F abundances in the barium star HD 123396
}

\author{
A. Alves-Brito ${ }^{1,2}$, A. I. Karakas ${ }^{3}$, D. Yong ${ }^{3}$, J. Meléndez ${ }^{4}$, and S. Vásquez ${ }^{1}$ \\ 1 Departamento de Astronomía y Astrofísica, Pontificia Universidad Católica de Chile, Av. Vicuña Mackenna 4860, Santiago, Chile \\ e-mail: abrito@astro.puc.cl \\ 2 Centre for Astrophysics and Supercomputing, Swinburne University of Technology, Hawthorn, Victoria 3122, Australia \\ 3 Research School of Astronomy and Astrophysics, The Australian National University, Cotter Road, Weston, ACT 2611, Australia \\ ${ }^{4}$ Universidade de São Paulo, IAG, Rua do Matão 1226, Cidade Universitária, São Paulo 05508-900, Brazil
}

Received 28 January 2001 / Accepted 15 July 2011

\begin{abstract}
Context. Barium stars are moderately rare, chemically peculiar objects, which are believed to be the result of the pollution of an otherwise normal star by material from an evolved companion on the asymptotic giant branch (AGB).

Aims. We aim to derive carbon, nitrogen, oxygen, and fluorine abundances for the first time from the infrared spectra of the barium red giant star HD 123396 to quantitatively test AGB nucleosynthesis models for producing barium stars via mass accretion.

Methods. High-resolution and high $S / N$ infrared spectra were obtained using the Phoenix spectrograph mounted at the Gemini South telescope. The abundances were obtained through spectrum synthesis of individual atomic and molecular lines, using the MOOG stellar line analysis program, together with Kurucz's stellar atmosphere models. The analysis was classical, using 1D stellar models and spectral synthesis under the assumption of local thermodynamic equilibrium.

Results. We confirm that HD 123396 is a metal-deficient barium $\operatorname{star}([\mathrm{Fe} / \mathrm{H}]=-1.05)$, with $A(\mathrm{C})=7.88, A(\mathrm{~N})=6.65, A(\mathrm{O})=7.93$, and $A(\mathrm{Na})=5.28$ on a logarithmic scale where $A(\mathrm{H})=12$, leading to $[(\mathrm{C}+\mathrm{N}) / \mathrm{Fe}] \approx 0.5$. The $A(\mathrm{CNO})$ group, as well as the $A(\mathrm{Na})$ abundances, is in excellent agreement with those previously derived for this star using high-resolution optical data. We also found $A(\mathrm{~F})=4.16$, which implies $[\mathrm{F} / \mathrm{O}]=0.39$, a value that is substantially higher than the $\mathrm{F}$ abundances measured in globular clusters of a similar metallicity, noting that there are no F measurements in field stars of comparable metallicity.

Conclusions. The observed abundance pattern of the light elements (CNO, F, and Na) recovered here as well as the heavy elements ( $s$-process) studied elsewhere suggest that the surface composition of HD 123396 is well fitted by the predicted abundance pattern of a $1.5 M_{\odot}$ AGB model star with $Z=0.001$. Thus, the AGB mass transfer hypothesis offers a quantitatively viable framework.
\end{abstract}

Key words. stars: AGB and post-AGB - stars: abundances - nuclear reactions, nucleosynthesis, abundances - stars: atmospheres stars: individual: HD 123396 (HIP 69834)

\section{Introduction}

Barium stars are chemically peculiar objects - dwarfs, subgiants, and giants - that show an excess of carbon and $s$-process elements in their atmosphere (e.g. Tomkin et al. 1989; Barbuy et al. 1992; Allen \& Barbuy 2006; Smiljanic et al. 2007). Identification of stars showing strong enhancements of neutron capture elements that can be produced by the slow neutron capture process (the $s$-process), as well as prominent $\mathrm{CH}, \mathrm{CN}$, and $\mathrm{C}_{2}$ molecular lines, dates back to Bidelman \& Keenan (1951). Since then, many other authors have sought to measure abundances of different species in order to better constrain the possible mechanisms by which barium stars can be formed.

The first detailed analysis of the barium star HD 46407 was by Buridge \& Burbidge (1957), who found overabundances of barium and many other elements relative to solar or normal giants. Carbon, nitrogen, and oxygen were first analysed in barium stars by Tomkin \& Lambert (1979, HR 774) and Smith (1984, $\zeta$ Cap), who demonstrated that $\mathrm{C}$ were roughly solar and $\mathrm{O}$ slightly deficient by 0.1 dex. Nitrogen, on the other hand, was found to be enhanced by 0.3 dex with respect to the Sun in the barium stars analysed. More recently, Allen \& Barbuy (2006) and Allen \& Porto de Mello (2007) acquired high-resolution spectra of giant and dwarf barium stars and obtained chemical abundances for several heavy elements ( $\mathrm{Sr}, \mathrm{Y}, \mathrm{Zr}, \mathrm{Ru}, \mathrm{Ba}, \mathrm{La}$, $\mathrm{Ce}, \mathrm{Pr}, \mathrm{Nd}, \mathrm{Sm}, \mathrm{Hf}, \mathrm{Pb}$ ), that are produced by neutron-capture nucleosynthesis (the $s$ and $r$ processes, see e.g., Sneden et al. 2008). In summary, detailed abundance analyses of the barium stars reveal that the heavy elements produced by the $s$-process are enhanced by factors of 2-30 with respect to normal giants, whereas $\mathrm{C}$ is overabundant by a factor of 3 in the most extreme cases. While barium and $\mathrm{CH}$ stars likely had mass transferred from a former AGB companion, the latter group show strong $\mathrm{C}_{2}$ molecular bands and higher $\mathrm{C} / \mathrm{O}$ abundance ratios when compared to the former (e.g., Luck \& Bond 1991; McClure 1997). The general view is that $\mathrm{CH}$ stars are more metal poor than barium stars, though there is no clear boundary.

The slow neutron capture process occurs in the advanced thermally-pulsing asymptotic giant branch (TP-AGB) phase of low and intermediate-mass stars (e.g., Busso et al. 1999). For this reason significant enrichment of $s$-process elements are not expected in stars in earlier evolutionary phases. The discovery that all observed barium stars belong to spectroscopic binary systems (Böhm-Vitense 1980; McClure et al. 1980; McClure 1983, 1984; Böhm-Vitense \& Johnson 1985; Jorissen \& Mayor 1988; McClure \& Woodsworth 1990; Udry et al. 1998a,b) with eccentricities significantly lower than a sample of spectroscopically normal G and K giants (McClure \& Woodsworth 1990; Jorissen et al. 1998) is evidence that the C and large $s$-process overabundances are the result of accretion of enriched material from a former TP-AGB companion rather than the result of internal nucleosynthesis. The accretion of material is the direct result 
of mass transfer via Roche Lobe overflow or stellar winds, and the low eccentricities arise from tidal circularization (Boffin \& Jorissen 1988; Han et al. 1995; Karakas et al. 2000; Izzard et al. 2010). In this context, CNO elements, along with $s$-process elements, are key tracers of the nucleosynthesis in the previous AGB progenitor, so they provide crucial constraints on the formation mechanism. According to the mass-transfer hypothesis, the former AGB stars would now be optically invisible white dwarfs, and several investigations with the IUE satellite have found the characteristic UV excesses typical of white dwarfs (e.g. Böhm-Vitense et al. 2000a,b).

Another unique tracer of AGB nucleosynthesis is the element F. Outside of the solar system, F abundances were first determined in the pioneering work of Jorissen et al. (1992) who analysed the correlation between $\mathrm{F}$ and $\mathrm{C}$ abundances in a sample of AGB stars. While it was clear from that study that $\mathrm{F}$ can be synthesized in AGB stars, and it is produced in the He-intershell along with $\mathrm{C}$ and $s$-process elements (Jorissen et al. 1992; Lugaro et al. 2004), the nucleosynthetic origin of F in the Galaxy is still somewhat of a mystery. To date, $F$ abundances have been determined for giant stars in the Galactic and extragalactic field (e.g., Jorissen et al. 1992; Cunha \& Smith 2005; Pandey 2006; Schuler et al. 2007; Pandey et al. 2008; Cunha et al. 2008; Uttenthaler et al. 2008; Abia et al. 2010), in Galactic and extragalactic globular clusters (Cunha et al. 2003; Smith et al. 2005; Yong et al. 2008; Lebzelter et al. 2008), and in postAGB stars and planetary nebulae (Werner et al. 2005; Zhang \& Liu 2005; Otsuka et al. 2008). For barium stars in particular, $\mathrm{F}$ abundances have been measured for only two stars (Jorissen et al. 1992) and thus much work is required on both the theoretical and observational sides to improve not only our knowledge of the nature of barium stars but also the astrophysical environment under which $\mathrm{F}$ is synthesized.

HD 123396 is of particular interest because it has an iron abundance of $[\mathrm{Fe} / \mathrm{H}]^{1} \approx-1$, and is the most metal-deficient barium star from the Allen \& Barbuy (2006) sample. The low metallicity of HD 123396 makes it unique among barium stars as it is right at the low end of the barium star metallicity distribution, and at a metallicity that encompasses the metal-rich tail of the halo, as well as the metal-poor tail of the disk (e.g., Luck \& Bond 1991; Junqueira \& Pereira 2001; Allen \& Barbuy 2006).

The binary nature of HD 123396 has not been definitively established. There is no evidence, as yet, for radial velocity variations. Jorissen et al. (2005) employed an additional check for binarity involving the comparison between the Hipparcos and Tycho-2 proper motions. However, this technique has been successfully employed for stars with parallaxes higher than 5 mas, while HD 123396 has a parallax of $1.78 \pm 0.73$ mas (van Leeuwen 2007).

Here in this study we present the first abundance estimates of C, N, O, and F for HD 123396 using spectra taken at infrared wavelengths using the high-resolution, near-infrared Phoenix spectrograph at Gemini-South. HD 123396 exhibits overabundances of heavy elements at the three $s$-element peaks: the light $s$-peak, which includes the elements $\mathrm{Sr}, \mathrm{Y}$, and $\mathrm{Zr}$, and denoted by the ratio $[l s / \mathrm{Fe}]$; the heavy $s$-peak, which includes $\mathrm{Ba}, \mathrm{Ce}$, $\mathrm{Nd}$, and $\mathrm{Sm}$, and is denoted by the ratio $[h s / \mathrm{Fe}]$; and the ratio of $[\mathrm{Pb} / \mathrm{Fe}]$. This makes HD 123396 an excellent tracer of chemically peculiar objects at low metallicities. The abundances of

\footnotetext{
1 We have adopted the usual spectroscopic notation, where $A(\mathrm{X})=$ $\log [n(\mathrm{X}) / n(\mathrm{H})]+12$ and $[\mathrm{X} / \mathrm{Y}]=\log [n(\mathrm{X}) / n(\mathrm{Y})]_{*}-\log [n(\mathrm{X}) / n(\mathrm{Y})]_{\odot}$, where $n(\mathrm{X})$ and $n(\mathrm{Y})$ are the number particle density of "X" and "Y" respectively.
}

the $\mathrm{CNO}$ elements and $\mathrm{F}$ need to be considered together with the $s$-process elements, because in the context of the AGB mass transfer hypothesis, $\mathrm{C}$ and $\mathrm{F}$ are produced together with the heavy elements.

\section{Observations}

We acquired high-resolution and high signal-to-noise $(S / N)$ spectra of HD $123396\left(\alpha=14^{\mathrm{h}} 17^{\mathrm{m}} 33.239, \delta=\right.$ $-83^{\circ} 32^{\mathrm{m}} 46^{\mathrm{s}}$.67: J2000) using the spectrograph Phoenix (Hinkle et al. 2003) on the Gemini-South $8 \mathrm{~m}$ telescope at Cerro Pachon, Chile, in 2009 June, under project GS-2009A-Q-26. The star was selected from the high-resolution optical abundance analysis carried out by Allen \& Barbuy (2006).

HD 123396 was observed in service mode using basically the same setup described in Yong et al. (2008), which allowed us to cover $1551 \leq \lambda \leq 1558 \mathrm{~nm}$ in the $H$ band and $2330 \leq$ $\lambda \leq 2340 \mathrm{~nm}$ in the $K$ band, both employing a slit width-size of 0.34 arcsec in order to reach a spectral resolution of $R=50000$. Four exposures of HD 123396 of $51 \mathrm{~s}$ each were taken at two different positions along the slit in the $H$ band $(H=6.3 \mathrm{mag})$ and of $80 \mathrm{~s}$ each in the $K$ band $\left(K_{\mathrm{s}}=6.1 \mathrm{mag}\right)$. The individual spectra in each band were co-added leading to $S / N=200$ in each band. The spectra were reduced with IRAF $^{2}$ employing standard procedures, which included dark and sky subtraction, flatfield correction, spectrum extraction, wavelength calibration, and telluric correction.

\section{Analysis}

To calculate reliable chemical abundances, it is necessary to obtain precise stellar atmospheric parameters such as temperature $\left(T_{\text {eff }}\right)$, surface gravity $(\log g)$, microturbulent velocity $(\xi)$ and metallicity $([\mathrm{Fe} / \mathrm{H}])$. We assumed a typical mass of $0.8 M_{\odot}$ for the star HD 123396 (e.g., VandenBerg et al. 2000). Using optical data, Allen \& Barbuy (2006) obtained photometric and spectroscopic parameters for this star. While the photometric parameters were obtained from optical and infrared colours by employing different temperature calibrations (e.g., Alonso et al. 1999; Ramírez \& Meléndez 2004) and classical equations of stellar evolution, the spectroscopic parameters were calculated by imposing excitation (temperature) and ionization (gravity) equilibrium of Fe I and Fe II lines. Here we have adopted the values given in Allen \& Barbuy (2006) as our first guess. However, using our own infrared data, the final atmospheric parameters (basically $T_{\text {eff }}$ and $[\mathrm{Fe} / \mathrm{H}]$ ) were iteratively adjusted (see Table 1).

Our abundance calculations were carried out using Kurucz model atmospheres (Castelli et al. 1997), the local thermodynamic equilibrium (LTE) spectral synthesis program MOOG (Sneden 1973), and the same line list presented and described in previous studies (e.g., Meléndez et al. 2003, 2008; Yong et al. 2008, for more details).

For consistency, we have checked the line list in both $H$ and $K$ bands by fitting the Arcturus spectrum (Hinkle et al. 1995, $R=100000)$ with CNO abundances from Ryde et al. (2010) and with $\mathrm{F}$ and $\mathrm{Na}$ abundances as given in Cunha et al. (2003). A macroturbulent velocity of $3.7 \mathrm{~km} \mathrm{~s}^{-1}$ was adopted (Ryde et al. 2010). For HD 123396, we then derived chemical

2 IRAF is distributed by the National Optical Astronomy Observatory,
which is operated by the Association of Universities for Research in
Astronomy (AURA) under cooperative agreement with the National Science Foundation. 


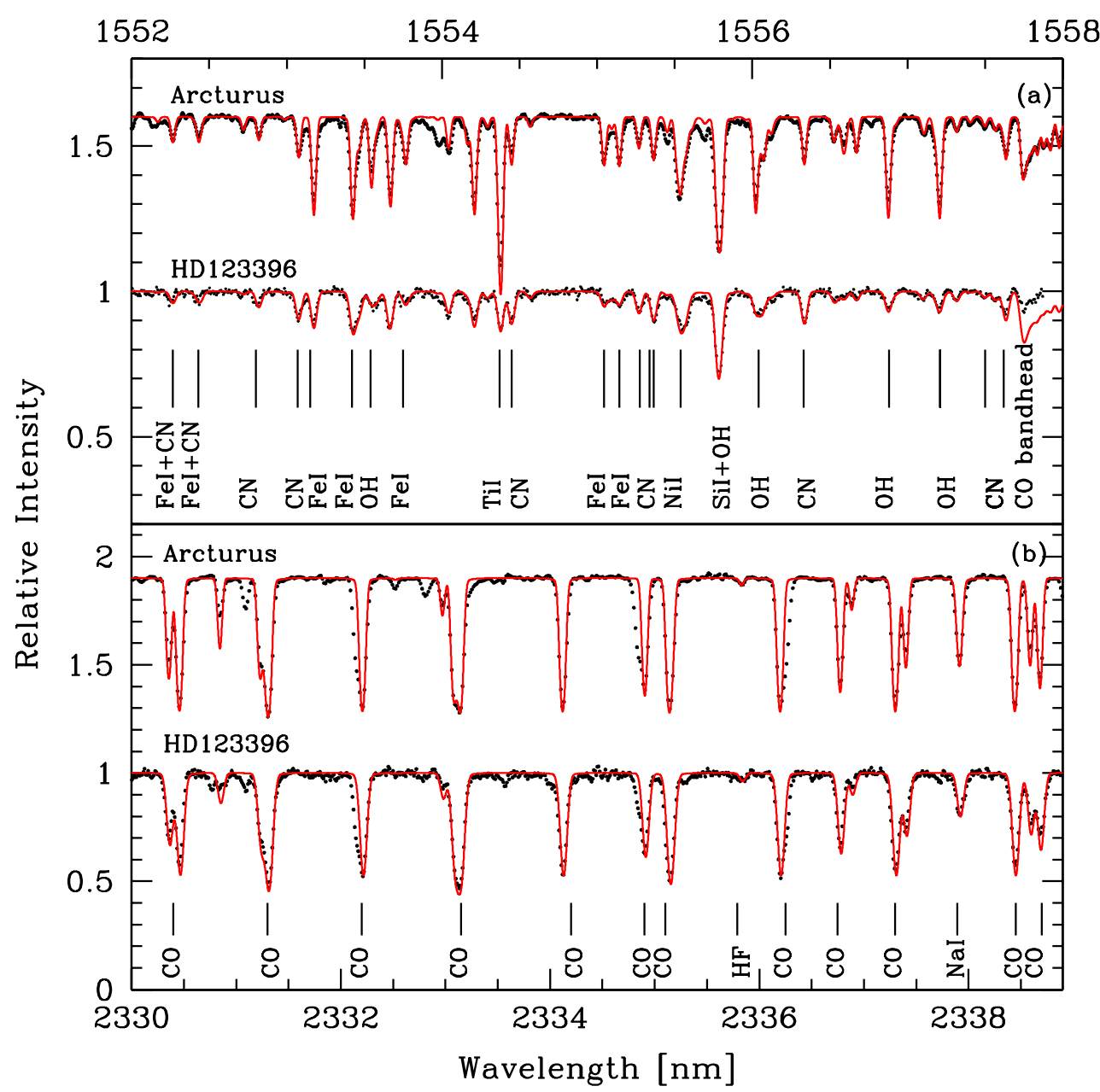

Fig. 1. Observed (points) and synthetic (lines) spectra of Arcturus and HD 123396 in the $H$ a) and $K$ b) bands. Several atomic and molecular lines are identified. Refer to the text for more details.

Table 1. Stellar parameters for HD 123396 and two barium stars from the literature with previous $\mathrm{F}$ abundance determinations.

\begin{tabular}{|c|c|c|c|c|c|c|c|}
\hline Star & $\begin{array}{l}T_{\text {eff }} \\
{[\mathrm{K}]}\end{array}$ & $\begin{array}{l}\log g \\
{[\operatorname{dex}]}\end{array}$ & $\begin{array}{c}\xi \\
{\left[\mathrm{km} \mathrm{s}^{-1}\right]}\end{array}$ & $\begin{array}{c}v_{\mathrm{r}} \\
{\left[\mathrm{km} \mathrm{s}^{-1}\right]}\end{array}$ & $\begin{array}{c}{[\mathrm{Fe} \mathrm{I} / \mathrm{H}]} \\
{[\mathrm{dex}]}\end{array}$ & $\begin{array}{c}{[\mathrm{Fe} \mathrm{II} / \mathrm{H}]} \\
{[\mathrm{dex}]}\end{array}$ & $\mathrm{Re}$ \\
\hline \multirow[t]{3}{*}{ HD 123396} & 4400 & 1.40 & 1.20 & 26.40 & -1.05 & & $(1)$ \\
\hline & 4360 & 1.40 & 1.20 & 26.64 & -1.19 & -0.99 & (2) \\
\hline & 4480 & 1.20 & 1.20 & 26.64 & -1.19 & -0.99 & (2) \\
\hline HD & 4200 & 0.80 & 2.50 & $\ldots$ & +0.05 & ... & (3) \\
\hline HD 178717 & 4300 & 1.00 & 2.20 & $\ldots$ & -0.18 & $\cdots$ & (3) \\
\hline
\end{tabular}

Notes. (1): this work; (2): Allen \& Barbuy (2006); (3): Smith (1984).

abundances of $\mathrm{C}, \mathrm{N}, \mathrm{O}, \mathrm{F}, \mathrm{Na}$, and $\mathrm{Fe}$ using $\mathrm{OH}, \mathrm{CO}$, and $\mathrm{CN}$ molecular bands and $\mathrm{Fe} \mathrm{I}$ lines in the $H$ band at $1555 \mathrm{~nm}$, as well as the HF (1-0) R9 line at $2335 \mathrm{~nm}(\chi=0.480 \mathrm{eV}$, $\log g f=-3.955 \mathrm{dex})$ and the NaI line at $2337 \mathrm{~nm}$ in the $K$ band. We note that the CO bandhead absorption at $1558 \mathrm{~nm}$ was discarded from the analysis. As seen in Fig. 1, the normalization at the extreme red edge of the spectrum makes the abundance fit very uncertain. Had we adjusted the $\mathrm{CO}$ molecular lines in the $H$ band, the $[\mathrm{C} / \mathrm{Fe}]$ inferred from the $\mathrm{CO}$ lines in the $H$ and $K$ bands would be different from the $\mathrm{CO}$ lines in the $K$ band yielding systematically higher abundances of $\mathrm{C}$ by 0.40 dex. To compute the synthetic spectra, we initially used the abundances derived by Allen \& Barbuy (2006) and then simultaneously changed $\mathrm{C}, \mathrm{N}$, and $\mathrm{O}$ abundances to obtain the best fits.
Table 2. Abundances for the star HD 123396 and two barium stars from the literature with previous $\mathrm{F}$ abundance determinations.

\begin{tabular}{lccccccc}
\hline \hline Star & $A(\mathrm{C})$ & $A(\mathrm{~N})$ & $A(\mathrm{O})$ & $A(\mathrm{~F})$ & $A(\mathrm{Na})$ & {$[\mathrm{F} / \mathrm{O}]$} & Ref. \\
\hline HD 123396 & 7.88 & 6.65 & 7.93 & 4.16 & 5.28 & +0.39 & $(1)$ \\
& 7.83 & 6.68 & 7.97 & $\ldots$ & 5.26 & $\ldots$ & $(2)$ \\
HD 121447 & 8.70 & 8.40 & 8.80 & 5.35 & $\ldots$ & +0.71 & $(3,4)$ \\
HD 178717 & 8.60 & 8.50 & 8.70 & 5.24 & $\ldots$ & +0.70 & $(3,4)$ \\
\hline
\end{tabular}

Notes. (1): this work; (2): Allen \& Barbuy (2006); (3, 4): Smith (1984); Jorissen et al. (1992). In this work, $A(\mathrm{C}, \mathrm{N}, \mathrm{O})_{\odot}=8.42$, 7.82, and 8.72; $A(\mathrm{~F}, \mathrm{Na}, \mathrm{Fe})_{\odot}=4.56,6.17$ and 7.50 (see Asplund et al. 2005; Meléndez et al. 2008). The [F/O] ratios from Ref. (4) were renormalized to our adopted solar abundances.

To take the instrumental profile and other broadening effects (e.g. macroturbulence) into account, the raw spectra were convolved with Gaussian functions of $F W H M \approx 0.40 \AA$ in the $H$ band and $F W H M \approx 0.60 \AA$ in the $K$ band. In Figs. 1 and 2 we show an example of spectral synthesis in the different bands.

\section{Results and discussion}

In Tables 1 and 2 we present the final atmospheric parameters and abundances derived for the star HD 123396. We also compare our results with those obtained by Allen \& Barbuy (2006) for the same star and for the two barium stars with 


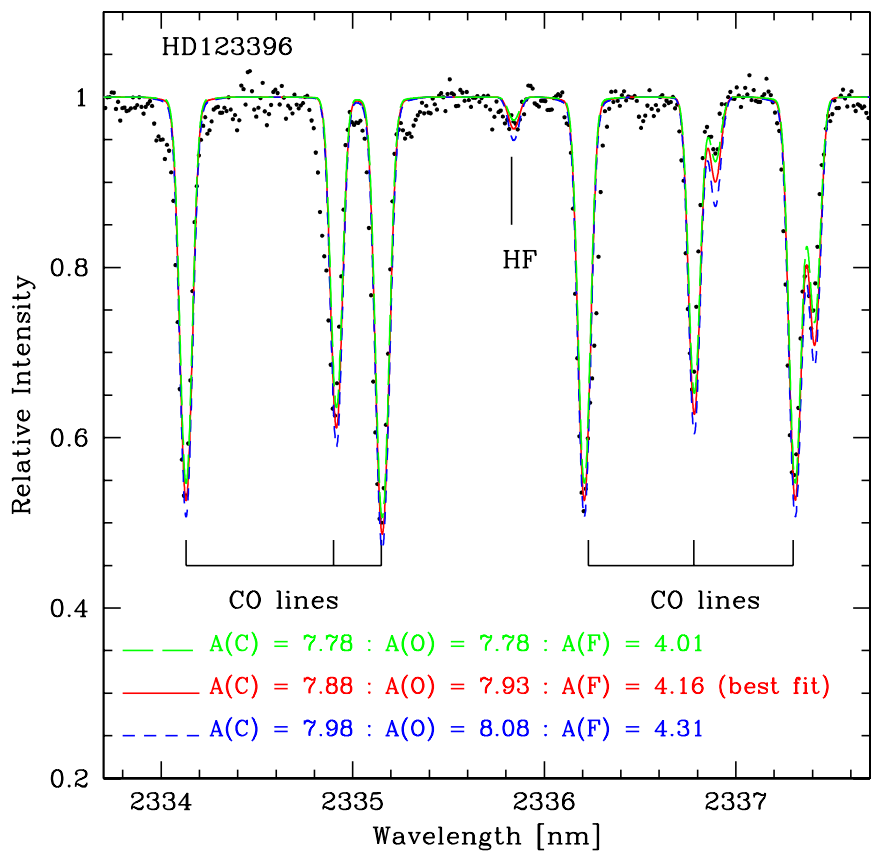

Fig. 2. Observed (points) and synthetic (line) spectra of HD 123396 for a sample of the wavelength regions in the $K$ band. The abundances derived for $\mathrm{C}, \mathrm{O}$, and $\mathrm{F}$ are labelled in the figure. The synthetic spectra stand for the best fit (solid line) and unsatisfactory ones (short and long dash lines) by varying the abundances of $\mathrm{C}$ by $\pm 0.10 \mathrm{dex}$, and $\mathrm{O}$ and $\mathrm{F}$ by \pm 0.15 dex, respectively (refer to the text).

F abundances previously derived by Jorissen et al. (1992). The primary source of errors in the abundances are the errors in the atmospheric parameters. Varying the atmospheric parameters by their typical uncertainties $\left(\Delta T_{\mathrm{eff}}= \pm 100 \mathrm{~K}, \Delta \log g= \pm 0.3 \mathrm{dex}\right.$, $\Delta \xi= \pm 0.2 \mathrm{~km} \mathrm{~s}^{-1}$ and $\left.\Delta[\mathrm{Fe} / \mathrm{H}]= \pm 0.1 \mathrm{dex}\right)$, we find that $\mathrm{C}, \mathrm{N}$, and $\mathrm{O}$ are uncertain by $0.10,0.12$, and 0.15 dex when all errors are added in quadrature. The $\mathrm{Na}$ and $\mathrm{F}$ abundances change by 0.05 dex and 0.15 dex, respectively. These values suggest that the total error in the $[\mathrm{F} / \mathrm{O}]$ ratio cancels out. In Fig. 1 we display an example of the spectral synthesis performed for the star in the $H$ and $K$ bands compared with that of Arcturus.

Using high-resolution optical spectra, Allen \& Barbuy (2006) performed a detailed abundance analysis of HD 123396 , yielding chemical abundances for $\mathrm{C}, \mathrm{N}, \alpha$, iron-peak, and neutron-capture elements. As can be seen in Table 1, we obtain $[\mathrm{Fe} / \mathrm{H}]=-1.05$ for this star, which is in excellent agreement with the mean value found by Allen \& Barbuy (2006), that is, $[\mathrm{Fe} / \mathrm{H}]=-1.09$. For the four other elements in common (see Table 2), we have found $\triangle A(\mathrm{C}, \mathrm{N}, \mathrm{O})=+0.05 \mathrm{dex}$, -0.03 dex and -0.04 dex, but $\Delta A(\mathrm{Na})=+0.02$ dex. While the differences in the absolute abundances are statistically insignificant, we recall that the $\mathrm{CNO}$ group elements are better studied in the infrared, where their lines are more numerous, and the continuum is formed deepest in the layers of the stellar atmosphere due to the opacity minimum of $\mathrm{H}^{-}$near the $H$ band. As presented in Table 7 of Barbuy et al. (1992), the $C$ abundances can vary up to +0.25 dex for the same star depending on the optical molecular lines used $\left(\mathrm{C}_{2}\right.$ or $\mathrm{CH}$ lines), likely from $3 \mathrm{D}$ and NLTE effects.

Using high-resolution infrared spectra acquired with the Fourier transform spectrometer on the Kitt Peak National Observatory's $4 \mathrm{~m}$ telescope, Jorissen et al. (1992) were the first to obtain $\mathrm{F}$ abundances for a large and heterogeneous sample of giant stars, which included two metal-rich barium stars. From
Table 2, one can see that HD 123396 has an [F/O] ratio that is $\sim+0.30$ dex lower than the two barium stars analysed by Jorissen et al. (1992). This result may not be surprising for the following reasons. First, the metallicity of HD 123396 is lower by roughly an order of magnitude than the other two barium stars (based on the iron abundances quoted by Smith 1984). Second, the recent analysis by Abia et al. (2010) resulted in significantly lower $\mathrm{F}$ abundances than in previous studies in the literature. The lower $\mathrm{F}$ abundances are associated with improved model atmospheres and atomic and molecular line lists in the $2.3 \mu \mathrm{m}$ region, and this has resulted in the identification of molecular blends.

Allen \& Barbuy (2006) show that HD 123396 is an $s$-process rich star (e.g., $[\mathrm{Pb} / \mathrm{Fe}]=1.00)$. Given that low and intermediatemass stars produce $s$-process elements during the AGB phase, and that barium stars are, generally, less evolved and not on the AGB leads to the conclusion that they obtained their heavyelement overabundances via mass transfer from a previous AGB companion. The high percentage of barium stars in wide binary systems is another piece of evidence for this hypothesis. Further evidence comes from the relatively high $\mathrm{F}$ abundance of HD 123396 that we derive in this work.

While the astrophysical site for F production in the Galaxy is uncertain with massive stars perhaps playing a dominant role (Meynet \& Arnould 2000; Cunha et al. 2003; Renda et al. 2004), there is strong evidence that $\mathrm{F}$ is produced in AGB stars (Jorissen et al. 1992; Abia et al. 2010). Possible reaction pathways for F production in AGB stars were outlined by Jorissen et al. (1992) and examined in detail by Forestini et al. (1992), Mowlavi et al. (1996), Lugaro et al. (2004), and Karakas et al. (2008). The F production in AGB stars occurs in the He-intershell via a complex series of proton, $\alpha$, and neutron-capture reactions, starting with the ${ }^{14} \mathrm{~N}(\alpha, \gamma){ }^{18} \mathrm{~F}\left(\beta^{+}\right){ }^{18} \mathrm{O}(p, \alpha){ }^{15} \mathrm{~N}(\alpha, \gamma){ }^{19} \mathrm{~F}$ reaction. The protons for the CNO cycle reaction ${ }^{18} \mathrm{O}(p, \alpha){ }^{15} \mathrm{~N}$ come from the ${ }^{14} \mathrm{~N}(n, p){ }^{14} \mathrm{C}$ reaction, which in turn requires free neutrons. ${ }^{14} \mathrm{~N}$ is one of the the main neutron poisons in AGB stellar models (see discussion in Busso et al. 1999). The ${ }^{13} \mathrm{C}(\alpha, n) \mathrm{O}^{16}$ reaction is the dominant source of free neutrons in low-mass AGB stars and is the main neutron source for the operation of the $s$ process. The ${ }^{22} \mathrm{Ne}(\alpha, n) \mathrm{Mg}^{25}$ neutron source also produces a small burst of neutrons during convective thermal pulses but models and observational evidence suggests that it plays a minor role (Busso et al. 2001; Abia et al. 2002). Fluorine can also be destroyed via He-shell burning via ${ }^{19} \mathrm{~F}(\alpha, p){ }^{22} \mathrm{Ne}$ at temperatures over about $300 \times 10^{6} \mathrm{~K}$, and $\mathrm{F}$ production has been shown to be a sensitive function of the initial mass and metallicity, with peak production occurring at $\approx 3 M_{\odot}$ at $Z=0.02$, which is reduced to $\approx 2 M_{\odot}$ at $Z=0.0001([\mathrm{Fe} / \mathrm{H}]=-2.3)$ (Lugaro et al. 2004; Karakas 2010). Furthermore, because F production occurs in the He-intershell, it is dredged to the surface via the repeated action of the third dredge-up, along with $\mathrm{C}$ and any $s$-process elements.

In Fig. 3a we show a plot of $[(\mathrm{C}+\mathrm{N}) / \mathrm{Fe}]$ vs. $[\mathrm{Fe} / \mathrm{H}]$ for normal giant stars from the literature (Meléndez et al. 2008; Ryde et al. 2010) compared with those obtained for barium stars in this work and from the literature (Smith 1984; Barbuy et al. 1992; Allen \& Barbuy 2006). This figure suggests that the $[(\mathrm{C}+\mathrm{N}) / \mathrm{Fe}]$ ratio we recover for HD 123396 lies on, or near, a linear extrapolation based on the more metal-rich barium stars, that is, $[(\mathrm{C}+\mathrm{N}) / \mathrm{Fe}] \approx 0.5$. On the other hand, normal $\mathrm{K}$ giant stars that have not experienced the first dredge up yet have $[(\mathrm{C}+\mathrm{N}) / \mathrm{Fe}] \approx 0$. We point out that some of the stars that were previously classified as the most metal-poor halo barium stars are now shown in fact to be $\mathrm{CH}$ or AGB stars (see e.g., Jorissen et al. 2005; Drake \& Pereira 2008; Pereira \& Drake 2009). However, barium stars present longer periods and greater 

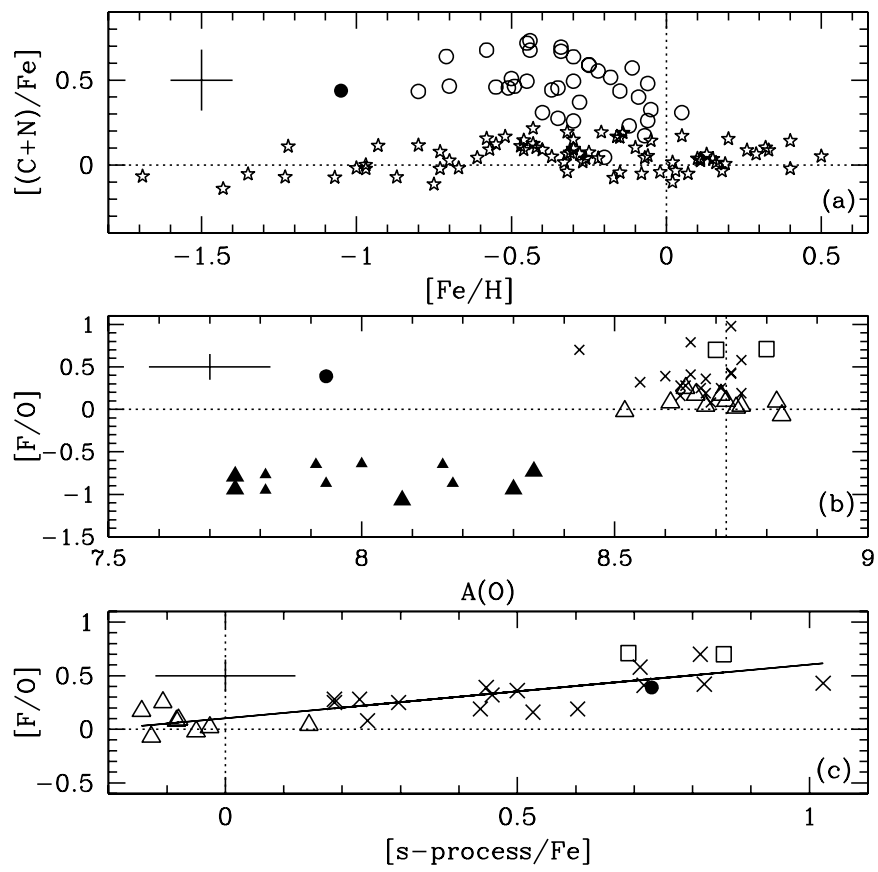

Fig. 3. a): $[(\mathrm{C}+\mathrm{N}) / \mathrm{Fe}]$ vs. $[\mathrm{Fe} / \mathrm{H}]$ for normal $\mathrm{K}$ giants (stars: Meléndez et al. 2008; Ryde et al. 2010) and barium stars in this work (filled circle) and from the literature (open circles: Smith 1984; Barbuy et al. 1992; Allen \& Barbuy 2006). b): [F/O] vs. A(O) for HD 123396 (filled circle) compared to (i) field stars - two barium stars (open squares: Jorissen et al. 1992), M giant stars (open triangles: Cunha et al. 2003), MS and S stars (crosses: Jorissen et al. 1992); and compared to (ii) GC's stars - M4 (small filled triangles: Smith et al. 2005) and NGC 6712 (large filled triangles: Yong et al. 2008). All abundances were renormalized to $A(\mathrm{~F})_{\odot}=4.56$ and $A(\mathrm{O})_{\odot}=8.72$. c) $:[\mathrm{F} / \mathrm{O}]$ vs. [s-process $\left./ \mathrm{Fe}\right]$, where $s$-process stand for $\mathrm{Y}, \mathrm{Zr}$ and $\mathrm{Nd}$. The $s$-process abundances were taken from Allen \& Barbuy (2006), Smith (1984, two barium stars), and Smith \& Lambert (1985, 1986, 1990, M, MS and $S$ stars). The dotted lines indicate the solar abundances. Typical uncertainties are quoted.

eccentricities than the $\mathrm{CH}$ stars (e.g. Vanture 1992a). From a chemical point of view, while classical barium and $\mathrm{CH}$ stars are $s$-process enriched to a similar degree, $\mathrm{CH}$ stars are differentiated by their strong $\mathrm{C}_{2}$ molecular bands, high $\mathrm{C} / \mathrm{O}$ abundances $([\mathrm{C} / \mathrm{O}] \geq 1)$ and $[(\mathrm{C}+\mathrm{N}) / \mathrm{Fe}] \approx+1.0$ (e.g. Vanture $1992 \mathrm{a}, \mathrm{b})$.

In Fig. 3b we plot $[\mathrm{F} / \mathrm{O}]$ vs. $A(\mathrm{O})$ for the star analysed in this work compared with other two disk barium stars, cool K, M, MS, and S giant stars from Jorissen et al. (1992) and Cunha et al. (2003), as well as stars from the GCs M4 and NGC 6712 (Smith et al. 2005; Yong et al. 2008), which have a metallicity similar to the barium star analysed in this work. For HD 123396, we find $[\mathrm{F} / \mathrm{O}]=+0.39$ dex, which means that, for the three barium stars analysed to date, $\langle[\mathrm{F} / \mathrm{O}]\rangle=+0.60 \pm 0.18$ dex. Interestingly, Smith et al. (2005) and Yong et al. (2008) report, respectively, $\langle[\mathrm{F} / \mathrm{O}]\rangle=-0.77 \pm 0.13 \operatorname{dex}(N=7$ stars $)$ and $\langle[\mathrm{F} / \mathrm{O}]\rangle=-0.89 \pm$ $0.13 \operatorname{dex}(N=5$ stars $)$ for the GCs M4 $([\mathrm{Fe} / \mathrm{H}]=-1.18)$ and NGC $6712([\mathrm{Fe} / \mathrm{H}]=-0.96)$. Our measurement of $\mathrm{F}$ in HD 123396 is the first measurement of $\mathrm{F}$ in a star whose metallicity, $A(\mathrm{O})$, overlaps with the GC data. From Fig. 3b one can infer that the $\mathrm{F} / \mathrm{O}$ ratio in HD 123396 is significantly different from the mean values found in the GCs studied, $\delta(\mathrm{F} / \mathrm{O}) \simeq 1.2$. This difference corresponds to $\sim 10-\sigma$ or a factor of $\sim 16$ higher than the uncertainty estimated above for the [F/O] ratio of HD 123396. In addition, the GC stars and HD 123396 are all metal-poor giants such that dwarf/giant systematic errors cannot be invoked to explain the abundance difference. However, HD 123396 is a

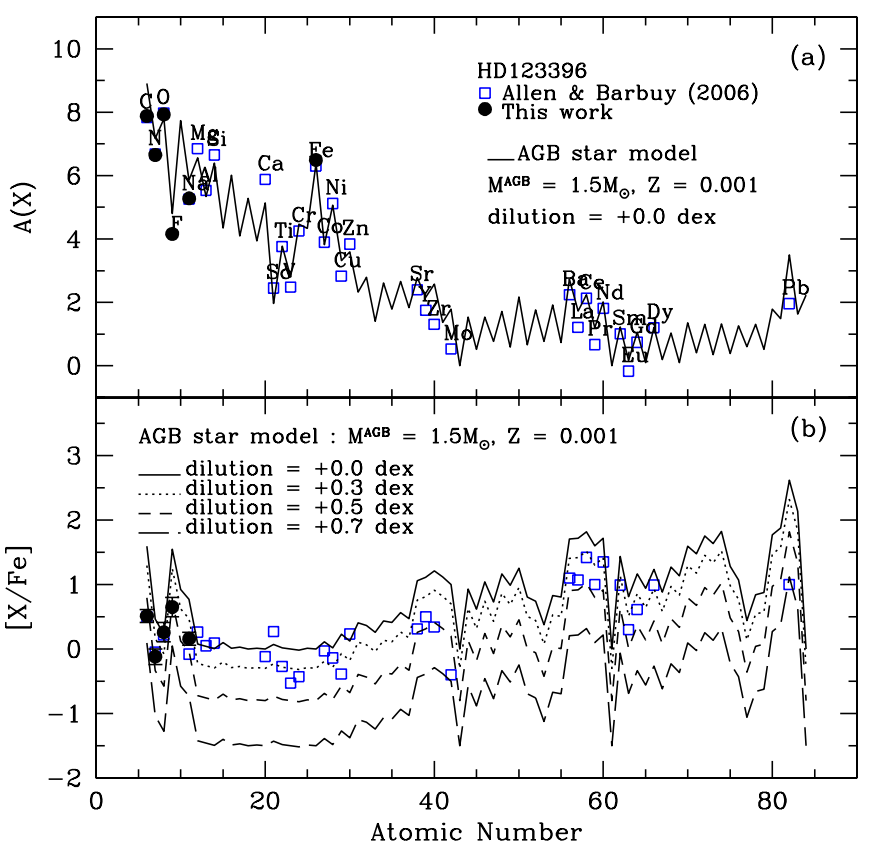

Fig. 4. The barium giant star HD 123396 observed abundance pattern compared with theoretical predictions of a $1.5 M_{\odot}$ AGB star of $Z=$ 0.001. Symbols and details on the model used are given in the figure and text.

chemically peculiar barium star that probably received its $\mathrm{F}$ from AGB mass transfer. According to this hypothesis, barium stars will likely have enhanced $\mathrm{F}$ abundances relative to normal field stars of the same metallicity. Thus, additional $\mathrm{F}$ measurements in metal-poor field stars are necessary to test if there is a real discrepancy in $\mathrm{F}$ abundances measured in normal field stars and GCs at such metallicity.

In Fig. 3c we present the $[\mathrm{F} / \mathrm{O}]$ abundances as a function of the mean abundances of two light s-process elements from the first $s$-element peak ( $\mathrm{Y}$ and $\mathrm{Sr}$ ) and one from the second $s$-process peak (Nd). The abundances were taken from different sources (Smith \& Lambert 1985, 1986, 1990; Allen \& Barbuy 2006), and we note that no attempt was made to take likely systematic effects into account. Clearly, HD 123396 follows the general trend for $[\mathrm{F} / \mathrm{O}]$ to increase with $s$-process elements as found by Jorissen et al. (1992). From a linear fit to the data it turns out that the scatter in the data is $\approx 0.15 \mathrm{dex}$, which is likely due to the convolution of measurement uncertainties from individual studies and the errors introduced by an inhomogeneous sample.

In Fig. 4 we show the abundances derived for HD 123396 in this work and in Allen \& Barbuy (2006), along with nucleosynthesis predictions from a $1.5 M_{\odot}, Z=0.001$ AGB star model. The AGB model was calculated using the stellar evolution code described in detail by Karakas (2010, and references therein), which uses the Vassiliadis \& Wood (1993) mass-loss rate on the $\mathrm{AGB}$, and with the addition of $\mathrm{C}$ and $\mathrm{N}$-rich low-temperature opacities tables from Marigo \& Aringer (2009). The $s$-process abundance predictions were calculated using the nucleosynthesis code described in Karakas (2010) and Karakas \& Lugaro (2010), updated to use a full network of 320 species for elements from hydrogen through to bismuth, reaction rates taken from the JINA REACLIB library (Cyburt et al. 2010). Solar abundances of $\mathrm{C}, \mathrm{N}, \mathrm{O}, \mathrm{Ne}, \mathrm{Mg}, \mathrm{Si}, \mathrm{S}, \mathrm{Ar}$, and $\mathrm{Fe}$ are the pre-solar nebula values from Table 5 of Asplund et al. (2009); $\mathrm{F}$ is the meteoritic value of $A(\mathrm{~F})_{\odot}=4.42$ from Table 1 of the same paper 
Table 3. Predicted surface composition from a scaled-solar $1.5 M_{\odot}, Z=0.001$ star model at the tip of the AGB.

\begin{tabular}{lcccccccccc}
\hline \hline Pocket & $A(\mathrm{C})$ & $A(\mathrm{~N})$ & $A(\mathrm{O})$ & $A(\mathrm{~F})$ & {$[\mathrm{F} / \mathrm{O}]$} & {$[\mathrm{Y} / \mathrm{Fe}]$} & {$[\mathrm{Zr} / \mathrm{Fe}]$} & {$[\mathrm{Ba} / \mathrm{Fe}]$} & {$[\mathrm{Nd} / \mathrm{Fe}]$} & {$[\mathrm{Pb} / \mathrm{Fe}]$} \\
\hline Initial & 7.29 & 6.69 & 7.55 & 3.24 & 0.00 & 0.00 & 0.00 & 0.00 & 0.00 & 0.00 \\
\hline 0.002 & 8.91 & 7.17 & 7.79 & 4.81 & 1.32 & 1.11 & 1.21 & 1.70 & 1.71 & 2.61 \\
0.0004 & 8.94 & 7.17 & 7.74 & 4.59 & 1.16 & 0.51 & 0.60 & 1.05 & 1.07 & 1.96 \\
\hline
\end{tabular}

Notes. The $[\mathrm{Fe} / \mathrm{H}]$ of the model star is -1.18 , and initial abundances are given in the first row.

The $[\mathrm{F} / \mathrm{O}]$ and $[\mathrm{X} / \mathrm{Fe}]$ abundances ratios were normalized to the solar abundances from Asplund et al. (2009).

(chosen because it has a lower uncertainty), and for many of the elements heavier than $\mathrm{Fe}$ we use the meteoritic values for the solar abundances (e.g., $\mathrm{Ga}, \mathrm{Sr}, \mathrm{Eu}, \mathrm{Pb}$ ). In order to obtain an enrichment of $s$-process elements, an exponentially decaying abundance of protons is artificially introduced into the top layers of the He-intershell at the deepest extent of each third dredge-up episode. We do this in exactly the same manner as outlined in Lugaro et al. (2004) and Karakas (2010). This partially mixed zone is required in order to facilitate the formation of a so-called ${ }^{13} \mathrm{C}$ pocket, which allows neutrons to be released via the ${ }^{13} \mathrm{C}(\alpha, n) \mathrm{O}^{16}$ reaction (see Straniero et al. 1995; Gallino et al. 1998; Goriely \& Mowlavi 2000; Herwig 2005, for more details). The mass of the proton profile is a free parameter that we set as a constant mass. We adopt two choices for the extent of the partially mixed proton zone: 1) $0.002 M_{\odot}$ and 2) $0.0004 M_{\odot}$ (or a fifth of the mass of our standard choice). We discuss the implications of this choice below. In Table 3 we present the surface composition for a selection of elemental abundances for the two choices of the partially mixed zone. These abundances from the tip of the AGB and represent the undiluted AGB composition.

The $\mathrm{F}$ abundance of the model star at the tip of the AGB is between $[\mathrm{F} / \mathrm{O}]=1.16-1.32$ (depending on the mass of the partially-mixed zone assumed in the model, see Table 3 ). Using the dilution formula from Bisterzo et al. (2010, their Eq. (6)) we can estimate the amount by which the AGB surface composition has been diluted into the observed star's envelope. Larger dilution factors can indicate that only a small amount of material was transferred, and/or that the star is now a giant with a large convective envelope. To match the measured $\mathrm{F}$ abundance of $+0.60 \pm 0.18$, we need to dilute the predicted $[\mathrm{F} / \mathrm{O}]$ ratio of 1.16-1.32 (see Table 3) by $0.5-0.7$ dex, depending on the size of the partially mixed zone. We note that our simple approach does not take into account the fact both $\mathrm{O}$ and $\mathrm{F}$ vary between predictions and observations, and is used only for a qualitative comparison between AGB model predictions and the observed abundances. However, the dilution range of $0.5-0.7$ also produces a good match to the observed $s$-process abundance distribution (see below), with the exception of the $\mathrm{Pb}$ abundance. Diluting the $\mathrm{C}$ and $\mathrm{N}$ abundances by $0.5-0.7$ dex gives $[\mathrm{C} / \mathrm{Fe}]=$ $+0.89-1.09$, and $[\mathrm{N} / \mathrm{Fe}]=-0.24$ to -0.04 (with corresponding values of $A(\mathrm{C})=8.21-8.41$ and $A(\mathrm{~N})=6.48-6.68$, respectively). If we assume that HD 123396 has already experienced the first dredge-up (FDU) that takes place following core $\mathrm{H}$-exhaustion, then there would be further decreases in $\mathrm{C}$ followed by an increase in $\mathrm{N}$.

The low N abundance that we find for HD 123396 may therefore be explained by dilution, even if the star has gone through the FDU. Stars with masses less than $\approx 2 M_{\odot}$ are observed to experience further mixing of $\mathrm{Li}$ and $\mathrm{CN}$ processed material after the luminosity bump (Gilroy 1989; Charbonnel 1994; Lind et al. 2009). If our star has experienced such mixing then there should be corresponding reductions in $\mathrm{C}$ and the ${ }^{12} \mathrm{C} /{ }^{13} \mathrm{C}$ ratio to $\approx 5-10$, followed by an increase in $\mathrm{N}$. The FDU predicts no change to the elemental $\mathrm{O}$ abundance, and the predictions from the AGB model are $[\mathrm{O} / \mathrm{Fe}]=0.22$ prior to dilution and $[\mathrm{O} / \mathrm{Fe}]=-0.48$ to -0.28 after. However, due to its low metallicity the star was probably alpha-enhanced initially, resulting in $[\mathrm{O} / \mathrm{Fe}]=0$ after dilution.

In terms of neutron-capture abundances, the model with our standard partially mixed proton mass of $0.002 M_{\odot}$ gives a predicted value of $[(\mathrm{Y}+\mathrm{Zr}+\mathrm{Nd}) / \mathrm{Fe}] / 3=1.34$ dex. After dilution of $0.50-0.70$ dex this yields $0.64-0.84$. A dilution of 0.60 dex matches the Allen \& Barbuy (2006) data point reasonably well, and gives $[\mathrm{F} / \mathrm{O}]=0.72$. To match the abundances of the observed $[l s / \mathrm{Fe}]$ and $[h s / \mathrm{Fe}]$ ratios given by Allen \& Barbuy (2006), it is necessary to use a lower dilution factor of 0.30 dex. On the other hand, a dilution factor greater than +1.0 dex is required to reduce the high predicted $\mathrm{Pb}$ abundance of $[\mathrm{Pb} / \mathrm{Fe}]=2.61$ to wthat is observed (1.20). Ignoring uncertainties in neutron-capture cross sections, the main factors affecting the production of $s$-process elements in low-mass AGB stars are the metallicity of the star, the stellar mass, and the size of the ${ }^{13} \mathrm{C}$ pocket (see discussion in e.g., Bisterzo et al. 2010). We can experiment with reducing the mass of the proton profile to show the effect of one of these uncertainties. We have calculated one model where we reduced the mass of the proton profile by a factor of $5\left(0.0004 M_{\odot}\right)$ and obtain $[(\mathrm{Y}+\mathrm{Zr}+\mathrm{Nd}) / \mathrm{Fe}] / 3=0.73$ and $[\mathrm{Pb} / \mathrm{Fe}]=1.96$. Here a dilution factor of $0.5-0.7$ dex gives $[(\mathrm{Y}+\mathrm{Zr}+\mathrm{Nd}) / \mathrm{Fe}] / 3$ between $0.03-0.23$, and $[\mathrm{Pb} / \mathrm{Fe}]$ abundances between 1.26-1.46. While it is possible to match the observed $\mathrm{Pb}$ abundance with the model with a reduced ${ }^{13} \mathrm{C}$ pocket, it is now no longer possible to match the lighter neutron-capture abundances.

It may well be possible to fine tune other model parameters, such as stellar mass, composition, or ${ }^{13} \mathrm{C}$ pocket size, in order to obtain a better fit to the observed abundance distribution. For example, Husti et al. (2009) compare their AGB model predictions to $s$-process barium star abundances. They were able to provide a reasonable match to the composition of HD 123396 by assuming a $1.5 M_{\odot}$ star of $Z=0.001$ and using a ${ }^{13} \mathrm{C}$ pocket that was a factor of 4.5 times less than their standard, along with a dilution factor of 1.0 dex. Given the many uncertainties inherent in current AGB models including mass loss, convection, and the formation of ${ }^{13} \mathrm{C}$ pockets (we refer to Herwig 2005, for a discussion), we conclude that the AGB mass transfer scenario is shown to be quantitatively viable in explaining the light and heavy elements in this object.

\section{Concluding remarks}

Employing high-resolution and high $S / N$ data acquired with the Gemini Observatory Phoenix spectrograph, we have derived $\mathrm{CNO}, \mathrm{F}, \mathrm{Na}$, and $\mathrm{Fe}$ abundances in the metal-deficient barium red giant star HD 123396. F along with $\mathrm{C}, \mathrm{N}$, and s-process elements are important for constraining the formation processes of chemically peculiar objects. 
The detailed chemical abundance analysis performed here indicates that the nucleosynthetic history of HD 123396 is explained well in the context of the AGB mass transfer hypothesis. Furthermore, we derive, for the first time, [F/O] of a field star (despite its chemical peculiarity) in the regime spanned by the $\mathrm{GC}$ data $([\mathrm{Fe} / \mathrm{H}] \approx-1)$. Our results confirm that the $\mathrm{GC}$ and field stars have different values of $[\mathrm{F} / \mathrm{O}]$ at a given $A(\mathrm{O})$ abundance. However, gathering more data is vitally important in order not only to draw firm conclusions on the nucleosynthetic origin of $\mathrm{F}$ in the Galaxy, but also to investigate how chemically peculiar objects are formed and how the different stellar populations (field versus GCs) are chemically related.

Acknowledgements. A.A.B. acknowledges CNPq (PDE, 200227/2008-4) and FONDECYT (3100013) for financial support and Dr. S. Ryder, from the Australian Gemini Office, for his assistance during the Phase II definitions of our Gemini/Phoenix observations. J.M. acknowledges support from FAPESP (2010/50930-6), USP (Novos Docentes), and CNPq (Bolsa de produtividade). We are grateful to Drs. D. Allen and R. Gallino for kindly answering our questions on their results and to Dr. M. Lugaro for help in preparing the nuclear network used in the nucleosynthesis code. We are also grateful to an anonymous referee for detailed and useful comments on a previous version of the paper. The spectra were obtained as part of the programme GS-2009A-Q-26. Based on observations obtained at the Gemini Observatory, which is operated by the Association of Universities for Research in Astronomy, Inc., under a cooperative agreement with the NSF on behalf of the Gemini partnership: the National Science Foundation (United States), the Science and Technology Facilities Council (United Kingdom), the National Research Council (Canada), CONICYT (Chile), the Australian Research Council (Australia), Ministério da Ciência e Tecnologia (Brazil), and Ministerio de Ciencia, Tecnología e Innovación Productiva (Argentina)

\section{References}

Abia, C., Domínguez, I., Gallino, R., et al. 2002, ApJ, 579, 817

Abia, C., Cunha, K., Cristallo, S., et al. 2010, ApJ, 715, L94

Allen, D. M., \& Barbuy, B. 2006, A\&A, 454, 895

Allen, D. M., \& Porto de Mello, G. F. 2007, A\&A, 474, 221

Alonso, A., Arribas, S., \& Martínez-Roger, C. 1999, A\&AS, 140, 261

Asplund, M., Grevesse, N., \& Sauval, A. J. 2005, in Cosmic Abundances as

Records of Stellar Evolution and Nucleosynthesis, ed. T. G. Barnes III \&

F. N. Bash (Sao Francisco: ASP), ASP Conf. Ser., 336, 25

Asplund, M., Grevesse, N., Sauval, A. J., \& Scott, P. 2009, ARA\&A, 47, 481

Barbuy, B., Jorissen, A., Rossi, S. C. F., \& Arnould, M. 1992, A\&A, 262, 216

Bidelman, W. P., \& Keenan, P. C. 1951, ApJ, 114, 473

Bisterzo, S., Gallino, R., Straniero, O., Cristallo, S., \& Käppeler, F. 2010, MNRAS, 404, 1529

Boffin, H. M. J., \& Jorissen, A. 1988, A\&A, 205, 155

Böhm-Vitense, E. 1980, ApJ, 239, L79

Böhm-Vitense, E., \& Johnson, H. R. 1985, ApJ, 293, 288

Böhm-Vitense, E., Carpenter, K., Robinson, R., Ake, T., \& Brown, J. 2000a, ApJ, 533, 969

Böhm-Vitense, E., Carpenter, K. G., \& Robinson, R. D. 2000b, ApJ, 545, 992

Buridge, E. M., \& Burbidge, G. R. 1957, ApJ, 126, 357

Busso, M., Gallino, R., \& Wasserburg, G. J. 1999, ARA\&A, 37, 239

Busso, M., Gallino, R., Lambert, D. L., Travaglio, C., \& Smith, V. V. 2001, ApJ, 557,802

Castelli, F., Gratton, R. G., \& Kurucz, R. L. 1997, A\&A, 318, 841

Charbonnel, C. 1994, A\&A, 282, 811

Cunha, K., \& Smith, V. V. 2005, ApJ, 626, 425

Cunha, K., Smith, V. V., Lambert, D. L., \& Hinkle, K. H. 2003, AJ, 126, 1305

Cunha, K., Smith, V. V., \& Gibson, B. K. 2008, ApJ, 679, L17

Cyburt, R. H., Amthor, A. M., Ferguson, R., et al. 2010, ApJS, 189, 240

Drake, N. A., \& Pereira, C. B. 2008, AJ, 135, 1070

Forestini, M., Goriely, S., Jorissen, A., \& Arnould, M. 1992, A\&A, 261, 157
Gallino, R., Arlandini, C., Busso, M., et al. 1998, ApJ, 497, 388

Gilroy, K. K. 1989, ApJ, 347, 835

Goriely, S., \& Mowlavi, N. 2000, A\&A, 362, 599

Han, Z., Eggleton, P. P., Podsiadlowski, P., \& Tout, C. A. 1995, MNRAS, 277, 1443

Herwig, F. 2005, ARA\&A, 43, 435

Hinkle, K., Wallace, L., \& Livingston, W. 1995, Publ. Astron. Soc. Pac., 107, 1042

Hinkle, K. H., Blum, R. D., Joyce, R. R., et al. 2003, SPIE, 4834, 353

Husti, L., Gallino, R., Bisterzo, S., Straniero, O., \& Cristallo, S. 2009, PASA, 26, 176

Izzard, R. G., Dermine, T., \& Church, R. P. 2010, A\&A, 523, A10

Jorissen, A., \& Mayor, M. 1988, A\&A, 198, 187

Jorissen, A., Smith, V. V., \& Lambert, D. L. 1992, A\&A, 261, 164

Jorissen, A., Van Eck, S., Mayor, M., \& Udry, S. 1998, A\&A, 332, 877

Jorissen, A., Začs, L., Udry, S., Lindgren, H., \& Musaev, F. A. 2005, A\&A, 441, 1135

Junqueira, S., \& Pereira, C. B. 2001, AJ, 122, 360

Karakas, A. I. 2010, MNRAS, 403, 1413

Karakas, A. I., \& Lugaro, M. 2010, PASA, 27, 227

Karakas, A. I., Tout, C. A., \& Lattanzio, J. C. 2000, MNRAS, 316, 689

Karakas, A. I., Lee, H. Y., Lugaro, M., Görres, J., \& Wiescher, M. 2008, ApJ, 676,1254

Lebzelter, T., Lederer, M. T., Cristallo, S., et al. 2008, A\&A, 486, 511

Lind, K., Primas, F., Charbonnel, C., Grundahl, F., \& Asplund, M. 2009, A\&A, 503,545

Luck, R. E., \& Bond, H. E. 1991, ApJS, 77, 515

Lugaro, M., Ugalde, C., Karakas, A. I., et al. 2004, ApJ, 615, 934

Marigo, P., \& Aringer, B. 2009, A\&A, 508, 1539

McClure, R. D. 1983, ApJ, 268, 264

McClure, R. D. 1984, Publ. Astron. Soc. Pac., 96, 117

McClure, R. D. 1997, Publ. Astron. Soc. Pac., 109, 536

McClure, R. D., \& Woodsworth, A. W. 1990, ApJ, 352, 709

McClure, R. D., Fletcher, J. M., \& Nemec, J. M. 1980, ApJ, 238, L35

Meléndez, J., Barbuy, B., Bica, E., et al. 2003, A\&A, 411, 417

Meléndez, J., Asplund, M., Alves-Brito, A., et al. 2008, A\&A, 484, L21

Meynet, G., \& Arnould, M. 2000, A\&A, 355, 176

Mowlavi, N., Jorissen, A., \& Arnould, M. 1996, A\&A, 311, 803

Otsuka, M., Izumiura, H., Tajitsu, A., \& Hyung, S. 2008, ApJ, 682, L105

Pandey, G. 2006, ApJ, 648, 143

Pandey, G., Lambert, D. L., \& Rao, N. K. 2008, ApJ, 674, 1068

Pereira, C. B., \& Drake, N. A. 2009, A\&A, 496, 791

Ramírez, I., \& Meléndez, J. 2004, A\&A, 417, 301

Renda, A., Fenner, Y., Gibson, B. K., et al. 2004, MNRAS, 354, 575

Ryde, N., Gustafsson, B., Edvardsson, B., et al. 2010, A\&A, 509, A20

Schuler, S. C., Cunha, K., Smith, V. V., et al. 2007, ApJ, 667, L81

Smiljanic, R., Porto de Mello, G. F., \& da Silva, L. 2007, A\&A, 468, 679

Smith, V. V. 1984, A\&A, 132, 326

Smith, V. V., \& Lambert, D. L. 1985, ApJ, 294, 326

Smith, V. V., \& Lambert, D. L. 1986, ApJ, 311, 843

Smith, V. V., \& Lambert, D. L. 1990, ApJS, 72, 387

Smith, V. V., Cunha, K., Ivans, I. I., et al. 2005, ApJ, 633, 392

Sneden, C. A. 1973, Ph.D. Thesis, University of Texas at Austin

Sneden, C., Cowan, J. J., \& Gallino, R. 2008, ARA\&A, 46, 241

Straniero, O., Gallino, R., Busso, M., et al. 1995, ApJ, 440, L85

Tomkin, J., \& Lambert, D. L. 1979, ApJ, 227, 209

Tomkin, J., Lambert, D. L., Edvardsson, B., Gustafsson, B., \& Nissen, P. E. 1989, A\&A, 219, L15

Udry, S., Jorissen, A., Mayor, M., \& Van Eck, S. 1998a, A\&AS, 131, 25

Udry, S., Mayor, M., Van Eck, S., et al. 1998b, A\&AS, 131, 43

Uttenthaler, S., Aringer, B., Lebzelter, T., et al. 2008, ApJ, 682, 509

van Leeuwen, F. 2007, A\&A, 474, 653

VandenBerg, D. A., Swenson, F. J., Rogers, F. J., Iglesias, C. A., \& Alexander,

D. R. 2000, ApJ, 532, 430

Vanture, A. D. 1992a, AJ, 103, 2035

Vanture, A. D. 1992b, AJ, 104, 1986

Vassiliadis, E., \& Wood, P. R. 1993, ApJ, 413, 641

Werner, K., Rauch, T., \& Kruk, J. W. 2005, A\&A, 433, 641

Yong, D., Meléndez, J., Cunha, K., et al. 2008, ApJ, 689, 1020

Zhang, Y., \& Liu, X.-W. 2005, ApJ, 631, L61 